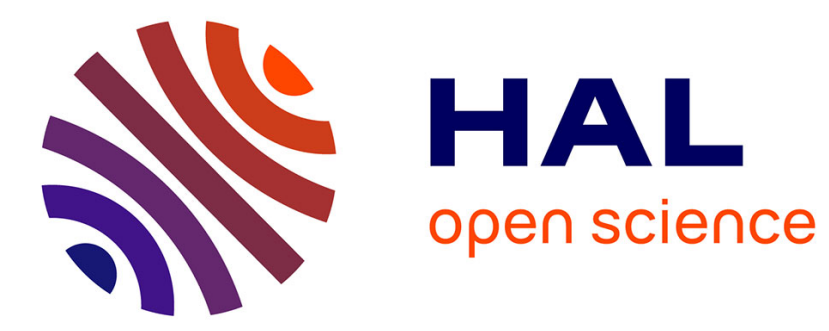

\title{
La phrase et le style: des invariants processuels à la variance individuelle
}

\author{
Stéphane Bikialo, Sabine Boucheron-Pétillon
}

\section{To cite this version:}

Stéphane Bikialo, Sabine Boucheron-Pétillon. La phrase et le style: des invariants processuels à la variance individuelle. Pratiques: linguistique, littérature, didactique, 2007, 135 (1), pp.177-193. 10.3406/prati.2007.2163 . hal-02306381

\section{HAL Id: hal-02306381 \\ https://hal.science/hal-02306381}

Submitted on 5 Oct 2019

HAL is a multi-disciplinary open access archive for the deposit and dissemination of scientific research documents, whether they are published or not. The documents may come from teaching and research institutions in France or abroad, or from public or private research centers.
L'archive ouverte pluridisciplinaire HAL, est destinée au dépôt et à la diffusion de documents scientifiques de niveau recherche, publiés ou non, émanant des établissements d'enseignement et de recherche français ou étrangers, des laboratoires publics ou privés. 


\section{La phrase et le style : des invariants processuels à la variance individuelle \\ Stéphane Bikialo, Sabine Boucheron-Pétillon}

\section{Résumé}

Dans le cadre de la production écrite, nous proposons une modélisation des pratiques de la phrase en fonction des quatre opérations d'écriture que sont - pour tout scripteur, novice ou expert - l'ajout, la suppression, la substitution et le déplacement comme des invariants processuels. Nous analysons tout particulièrement, dans une dynamique syntaxique, les praticiens de l'ajout et de la suppression, en la croisant avec l'élasticité du langage définie par Greimas. L'analyse des manuscrits de Leiris montre en effet qu'il relève de ce que Barthes appelle les « esprits à catalyse », tandis que Chateaubriand s'apparente aux « esprits à ellipse » - chacun travaillant, pour l'amplifier ou le réduire, le volume textuel du texte en devenir. L'objectif ultime de nos analyses est d'établir non pas une nouvelle définition du style d'auteur, mais de proposer des profils de praticiens de la phrase, profils appliqués aux textes littéraires (écrivains/ hyper-experts) comme à l'écriture non créative (écrivants/ experts).

\section{Citer ce document / Cite this document :}

Bikialo Stéphane, Boucheron-Pétillon Sabine. La phrase et le style : des invariants processuels à la variance individuelle. In: Pratiques : linguistique, littérature, didactique, n¹35-136, 2007. Le style en questions. pp. 177-193;

doi : https://doi.org/10.3406/prati.2007.2163

https://www.persee.fr/doc/prati_0338-2389_2007_num_135_1_2163

Fichier pdf généré le 13/07/2018 


\title{
La phrase et le style : des invariants processuels à la variance individuelle
}

\author{
Stéphane Bikialo \\ Université de Poitiers \\ Sabine Pétillon \\ Item-CNRS/ENS-UIm
}

A Jean-Pierre Seguin

"Le style, dame, tout le monde s'arrête devant, personne n'y vient à ce truc-là. Parce que c'est un boulot très dur." (Céline)

« La phrase est donc à chaque fois un événement différent; elle n'existe que dans l'instant où elle est proférée et s'efface aussitôt ; c'est un événement évanouissant ${ }^{(1)}$. Au sein de cet événement qu'est la phrase - mais aussi l'énonciation et l'énoncé dont elle est une des manifestations ${ }^{(2)}$ - le style forme une sorte d'«événement dans l'événement » selon la formule riche d'implications de L. Jenny ${ }^{(3)}$. Par cette mise en avant du style et de la phrase comme «événement», on privilégie évidemment leur conception dynamique. De fait, le style comme la phrase se définissent par leur dynamisme. A. Herschberg Pierrot y a récemment insisté pour le style, dans Le Style en mouvement (2005), qui se conclut ainsi : " Loin d'être une catégorie stable et homogène, le style apparaît bien plutôt comme un ensemble complexe de processus, une activité de transformation et de singularisation de l'œuvre» ${ }^{(4)}$. De même, E. Benveniste souligne ce dynamisme au sujet de la phrase : « le sens d'une phrase est autre chose que le sens des mots qui la composent », «la phrase, variété sans limite, est la vie même du langage en action » ${ }^{(5)}$.

(1) E. Benveniste, Problèmes de linguistique générale, Gallimard, 1974 : 227.

(2) Rappelons que pour M. Foucault (dans L'Archéologie du savoir, Gallimard, 1969 : 40), « un énoncé est toujours un événement » et l'analyse du champ discursif vise à "saisir l'énoncé dans l'étroitesse et la singularité de son événement ».

(3) L. Jenny, La Parole singulière, Belin, 1990 : 20. S. Bikialo a théorisé ce lien entre énonciation et style dans Plusieurs mots pour une chose. De la nomination multiple au style de Claude Simon, thèse de doctorat, Université de Poitiers, 2003, chapitre 1.

(4) A. Herschberg Pierrot, Le Style en mouvement, Belin, 2005: 181.

(5) E. Benveniste, $1974: 226$ et $1966: 129$. 
Cette approche dynamique - favorisée en particulier par l'apport de la dimension génétique permettant d'envisager une stylistique ou « poétique de l'écriture opposée à une poétique du texte ${ }^{(6)}$ - est la condition même du statut du style et de la phrase : concevoir en effet le style comme « processus continué » ${ }^{(7)}$ (de singularisation de l'œuvre) est une manière d'éviter l'approche discontinue, d'assemblage de procédés, souvent conçus comme s'écartant de la norme ; de la même façon, envisager la phrase comme dynamique revient - et Benveniste y insiste - à en faire l'unité du discours - ou mode sémantique - nécessitant une autre linguistique que la linguistique de la langue, du système.

Il s'agit ici de proposer une approche du style en production, en nous attachant plus particulièrement à la phrase et aux différents processus de genèse phrastique. Après avoir rendu compte rapidement des débats récents menés autour de la phrase, cadre à la fois ouvert et contraignant, nous montrerons que ce modèle de la phrase comme unité de contraintes doit intégrer la performance experte - observable dans les manuscrits de travail des écrivains - et que cette prise en compte permet de distinguer des profils de praticiens de la phrase, profils élaborés notamment à partir des deux dynamiques scripturaires que sont l'ellipse, figure de la condensation, et la catalyse, figure d'expansion. Ce faisant, nous replaçons nos analyses dans une problématique plus large et propre aux sciences du langage : celle de l'élasticité du langage définie par Greimas mais aussi celle de la variation, que nous abordons de façon dynamique en la transposant aux processus. Les quatre opérations d'écriture - ajout, suppression, substitution, déplacement - dont dispose tout scripteur sont ici abordées comme des invariants processuels autorisant des différences individuelles. Nous proposons donc une modélisation de la genèse phrastique qui rend compte à la fois des invariants opérationnels et des pratiques différentielles. Nous montrerons enfin comment émerge ce mouvement du style au sein de la phrase dans le manuscrit de Langage tangage de M. Leiris.

\section{La phrase : théorie et pratiques singulières}

\section{Production écrite et plan phrastique : un " modelage étroit de la pensée"}

Longtemps centrale en grammaire bien sûr, mais aussi sur le plan de l'apprentissage (ses vertus pédagogiques n'ont plus à faire leurs preuves, et chaque écolier sait en donner au moins une définition), la phrase a connu ces dernières décennies une véritable remise en cause, sous l'impulsion des travaux sur le français parlé, en linguistique textuelle et en pragmatique. Dans le champ des études sur le français parlé, les travaux de C. Blanche-Benveniste touchent un domaine macro-syntaxique dans lequel une relation de dépendance ou d'indépendance s'exerce entre deux segments, bien qu'il n'existe entre eux aucune relation de type rectionnel ${ }^{\left({ }^{(8)}\right.}$; de même, les recherches menées par M.-A. Morel et L. Danon-Boileau, partant du caractère inopérant de la phrase pour aborder l'oral, proposent la notion de « paragraphe intonatif», structuré - sur la base d'indices suprasegmentaux - en termes de " préambule », " rhème » et " postrhème ». En pragmatique, A. Berrendonner propose de dépasser la phrase pour s'attacher aux notions de «clause » et de «pé-

(6) R. Debray-Genette, "Génétique et poétique : esquisse de méthode », Littérature ${ }^{\circ} 28$, $1977: 20$.

(7) A. Herschberg Pierrot, $2005: 4$.

(8) C. Blanche-Benveniste, 1990. Les rappels qui suivent s'appuient en partie sur P. Le Goffic, 2005. 
riode ». Il fait « l'hypothèse qu'il existe un niveau auquel la chaîne parlée s'articule en unités minimales à fonction communicative », qu'il appelle « clauses ou énonciations » ${ }^{(9)}$ et qui sont définies non pas par rapport à la prédication ou à une modalité d'énonciation mais par leur fonction communicative et leur capacité à s'intégrer dans un rang de niveau supérieur : la période. En linguistique textuelle les travaux de B. Combettes et de J.-M. Adam ${ }^{(10)}$ ont montré qu'il convenait d'adopter une nouvelle terminologie adaptée à ce nouveau niveau de l'analyse linguistique qu'est le texte. Aussi diverses soient-elles, ces perspectives ont pour objectif de fonder une interface entre la phrase et le texte, entre la syntaxe et l'énonciation - d'où le développement, pour remplacer la phrase, des notions de «clause», «période», « séquence» ou encore « unité noyau». De façon également très pertinente, J.-M. Adam propose une typologie des niveaux d'analyse textuelle, de la texture à la structure : la proposition-énoncée se combine, grâce aux différents types de liage en périodes qui elles-mêmes constituent des séquences - le texte étant formé de séquences et délimité lui-même par le paratexte ${ }^{(11)}$.

Remise en cause, la phrase, concept fondateur émergé au XVIII ${ }^{\mathrm{e}}$ siècle comme l'a brillamment montré J.-P. Seguin, fait donc une entrée controversée dans le troisième millénaire ${ }^{(12)}$.

Or il se trouve que les recherche développées en psychologie cognitive depuis plus de vingt ans notamment, pour ce qui est de la production écrite, grâce aux modèles rédactionnels, d'une part, et celles que la linguistique génétique a menées sur les manuscrits de travail des écrivains, d'autre part, illustrent chacune à leur façon ce constat de Benveniste : «Nous communiquons par des phrases, même tronquées, embryonnaires, incomplètes, mais toujours par des phrases » ${ }^{(13)}$. Dans la dichotomie définie par Benveniste entre la langue comme sémiotique et la langue comme sémantique, cette citation a de quoi surprendre - le plan syntaxique (forme) étant en quelque sorte confondu ici avec le plan énonciatif (sens) -, sauf à considérer que la phrase et l'énoncé constituent deux réalisations d'une seule et même entité langagière. C'est précisément ce que Benveniste souligne : «Il nous semble qu'on doit tracer à travers la langue entière une ligne qui départage deux espèces et deux domaines du sens et de la forme, bien que, voilà encore un des paradoxes du langage, ce soient les mêmes éléments qu'on trouve de part et d'autre, dotés cependant d'un statut différent » ${ }^{(14)}$.

L'étude des manuscrits de travail des écrivains permet d'observer que 1'énonciation se construit dans le cadre de la phrase. En production écrite - qui est rigou-

(9) A. Berrendonner, $1990: 26$.

(10) A ce titre, voir la très éclairante mise au point opérée par B. Combettes sur les compétences textuelles dans «Phrase, texte, discours : quels cadres pour étudier la langue? », Pratiques, 107/108, 2000.

(11) J.-M. Adam, Linguistique textuelle. Des genres de discours aux textes, Nathan-université, 1999. L'auteur insiste sur la définition de la proposition énoncée comme unité minimale de la linguistique textuelle : «L'unité minimale que nous adopterons sera la proposition énoncée. Nous choisissons de l'appeler “proposition énoncée” pour souligner le fait qu'il s'agit d'une part, d'une unité résultant d'un acte d'énonciation, et, d'autre part, d'une unité liée, c'est-à-dire constituant un fait de discours et de textualité. Toute proposition comporte trois dimensions complémentaires auxquelles s'ajoute le fait qu'il n'y a pas de proposition isolée : même apparaissant seule, une proposition répond à une ou plusieurs autres et/ou en appelle implicitement une ou plusieurs autres », p. 50.

(12) J.-P. Seguin, 1993 et G. Kleiber, « Faut-il dire adieu à la phrase ? », L'information grammaticale, 98, $2003: 17-22$.

(13) E. Benveniste, $1974: 224$.

(14) Ibid. 
reusement notre domaine, le sens de la phrase - « le sens de la phrase est l'idée qu'elle exprime »-s'élabore par un «modelage étroit [de la pensée] dans la structure linguistique ${ }^{(15)}$, et ce modelage obéit évidemment à des contraintes formelles. Il n'y a pas d'une part les contraintes formelles de la grammaire et d'autre part - sans connexion entre les deux - le sens intenté délesté de son incarnation formelle. Au contraire, cet intenté émerge grâce à des essayages formels au cours desquels il est possible d'observer qu'un même contenu de pensée a revêtu des formes diverses : comme si la phrase ultime était la traduction finale de toutes les formes d'elle-même dont elle a fait l'essai avant de se fixer. Se fixer dans une forme ultime convenant au type de texte dans lequel elle évolue. C'est - à nos yeux - ce que le manuscrit de travail donne à voir. L'écriture en tant que processus est une rencontre médiatisée - par la plume, le clavier, etc. - entre un vouloir dire et des (im)possibles formels :

«Il faut tracer une distinction à l'intérieur du domaine sémantique entre la multiplicité indéfinie des phrases possibles, à la fois par leur diversité et par la possibilité qu'elles ont de s'engendrer les unes les autres, et le nombre toujours limité, non seulement de lexèmes utilisés comme mots, mais aussi des types de cadres syntaxiques auxquels le langage a nécessairement recours ${ }^{(16)}$.

Au moment d'écrire, tout scripteur se trouve tout à la fois en présence d'un « vouloir dire »-souvent relativement flou, voire inconscient - et devant cette "multiplicité indéfinie des phrases ». Si l'infini des possibles offerts - au moment même d'écrire - a donné lieu à un nombre important d'études, en psychologie développementale ainsi qu'en génétique et en didactique, ce que Benveniste appelle les « cadres syntaxiques » semble moins travaillé. Or

« il ne faut pas rejeter la phrase (concept puissant et nécessaire) comme concept organisateur central. [...] La syntaxe est un moyen et non une fin (moyen adaptable, révisable, violable), mais c'est un moyen contraignant ${ }^{(17)}$.

\section{Le travail de la phrase : un infini contraint}

La modélisation que nous proposons prend appui tout à la fois sur l'élasticité du langage et sur des données textuelles en production écrite observables dans les manuscrits de travail des écrivains. Elles sont bien sûr à vérifier auprès de scripteurs experts dans un programme de recherche à venir. La «multiplicité infinie des phrases possibles » qu'évoque Benveniste se retrouve dans le concept d'élasticité du discours développé par Greimas :

« [L'élasticité du discours] consiste dans l'aptitude du discours à mettre à plat, linéairement, des hiérarchies sémiotiques, à disposer en succession des segments discursifs relevant des niveaux divers d'une sémiotique donnée. La production du discours se trouve ainsi caractérisée par deux sortes d'activités apparemment contradictoires : l'expansion et la condensation. [...] ${ }^{(18)}$.

(15) «La conversion de la pensée en discours est assujettie à la structure formelle de l'idiome considéré, c'est-à-dire à une organisation typologique qui, selon la langue, fait tantôt prédominer le grammatical et tantôt le lexical ", Benveniste, $1974: 228$.

(16) Ibid. : 229.

(17) P. Le Goffic, $2005: 63$.

(18) L'auteur développe ensuite : «La prise en considération de l'élasticité du discours s'impose avec force en sémantique : on y constate, en effet, que des unités discursives de dimensions différentes peuvent être reconnues comme sémantiquement équivalentes. L'activité 
L'élasticité du discours ainsi que les procédés opposés d'expansion et de condensation (autorisés par cette élasticité) permettent qu'un contenu de sens équivalent puisse être exprimé par des unités discursives de taille et de complexité variables. Ainsi, pour résumer L'Odyssée, on peut tout aussi bien proposer une formule elliptique - «Ulysse rentre à la maison »-qu'une phrase plus expansée décrivant de façon relativement détaillée le retour du héros. Les différentes formulations pour un contenu identique sont rendues possibles en fonction de ce que la sémantique cognitive appelle le degré de spécificité (Langacker). C'est ce degré de spécificité qui oriente la formulation vers une forme elliptique (condensation) ou expansée (expansion). La variation possible entre formulation condensée $v s$ formulation expansée est formulée différemment par Stéphane Robert :

« Cette caractéristique particulière des langues, qui relève de la plasticité générale du langage, est permise par le fait qu'il existe un niveau supérieur d'organisation du sens et que le locuteur dispose, pour la construction de la représentation linguistique de ce sens, d'unités diverses associant forme et sens et dont la latitude combinatoire est effectivement remarquable ${ }^{(19)}$.

Dans un article consacré à «Flaubert et la phrase» (repris dans Nouveaux essais critiques), mais aussi dans ses cours (La Préparation au roman), Barthes revisite, sans le savoir, l'élasticité du discours décrite en sciences du langage, en s'appuyant sur les notions d' « ellipse » et de «catalyse » :

«L'ellipse est limitée par la structure même du langage. Cette même structure permet au contraire de donner libre cours, sans limite, aux corrections augmentatives; d'un côté les parties du discours peuvent être indéfiniment multipliées (ne serait-ce que par la digression), et de l'autre [...] la phrase peut être pourvue à l'infini d'incises et d'expansion : le travail catalytique est théoriquement infini ; même si la structure de la phrase est en fait réglée et limitée par des modèles littéraires $[\ldots]$ ou par des contraintes physiques [...], il n'en reste pas moins que l'écrivain, affronté à la phrase, éprouve la liberté infinie de la parole, telle qu'elle est inscrite dans la structure même du langage $»{ }^{(20)}$.

Il propose également une typologie sommaire des corrections opérées par les écrivains dans leurs manuscrits. Il distingue ainsi deux types principaux de corrections : les corrections substitutives (qui affectent l'axe vertical des paradigmes et le plan lexical) et les corrections prédicatives, qui touchent au volume syntaxique de la phrase et sont soit des corrections diminutives (ellipse), soit augmentatives (expansion, catalyse). L'ellipse et la catalyse sont abordées comme des processus scripturaires dynamiques opposés et que l'on peut regarder comme une formulation rhétorique du binôme expansion / condensation de Greimas. Barthes commentait ainsi un schéma opposant « ellipse » et «catalyse » dans la « Séance du 03 mars $1979 »$ de son cours au Collège de France :

«Entre le haïku et le récit, il y a ce mouvement, ce double mouvement, qui est si

métalinguistique, reconnaissable à l'intérieur du discours, et le phénomène de la paraphrase, considéré dans son principe, relèvent de cette élasticité du discours dont l'exemple le plus frappant est constitué par le jeu des dénominations (= condensations) et des définitions (= expansions) linguistiques. », dans A.-J. Greimas et J. Courtès, 1979 : 116-117.

(19) S. Robert, communication personnelle, recherches en cours.

(20) R. Barthes, «Flaubert et la phrase », Nouveaux essais critiques, Seuil, 1967 : 135. Les recherches de Barthes autour de la phrase ont donné lieu à un séminaire animé par J.-L Lebrave (2002-2004) de 1'équipe «Manuscrits et Linguistique » alors dirigée par A. Grésillon. 
important pour comprendre le problème théorique de l'énonciation ou, en tout cas, de la discursivité, double mouvement qu'on pourrait inscrire sous le nom d'une double figure de rhétorique : l'ellipse, qui est une figure de la condensation, et la catalyse, une figure de l'extension ${ }^{(21)}$.

Si l'élasticité du discours est établie, comme nous venons de le voir, à la fois en théorie linguistique (Greimas) et en rhétorique, c'est dans le cadre des études sur la production écrite qu'elle est le mieux explorée, notamment en linguistique génétique et en psychologie cognitive. Le parcours détaillé que nous venons de proposer a pour objectif non seulement de mettre en commun des problématiques proches mais aussi d'insister sur les frontières de l'objet que l'on cherche à saisir. Ici, plus particulièrement, l'expansion (catalyse) ou la condensation (ellipse) constituent des processus qui s'appliquent aussi bien à la phrase (définie comme une structure prédicative) qu' au texte comme matière dont la cohérence est en voie de constitution. Ces deux mécanismes ont pour fondement commun la plasticité du langage, qui est une propriété spécifique aux langues naturelles - une propriété que l'écriture (ou la scription dans une terminologie barthésienne) met en œuvre de façon dynamique.

\section{Genèses phrastiques : opérations d'écriture et variabilité individuelle}

Les mécanismes de l'expansion et de la condensation peuvent être observés dans les manuscrits d'écrivains; ils font partie du stock d'opérations d'écriture que le scripteur novice ou expert a - plus ou moins consciemment - à sa disposition.

L'étude des manuscrits de travail des écrivains permet de voir que la phrase est l'unité dans laquelle s'opère, à l'écrit, la répartition entre un contenu de base et des unités morpho-syntaxiques ${ }^{(22)}$. C'est donc au plan phrastique que s'opère le modelage de la pensée, pour reprendre la formule de Benveniste. Ce modelage se fait grâce aux opérations d'écriture que sont l'ajout, la substitution, le déplacement et la suppression. L'observation de ce modelage permet de souligner que si la phrase du texte imprimé est un continué de gauche à droite que la lecture active, il n'en va pas de même lors de sa genèse. En effet, une phrase en cours d'élaboration est susceptible d'être révisée - grâce aux opérations scripturaires - à tous les niveaux de dépendance (groupes ou propositions) et à tous les moments de sa genèse. Au moment même d'écrire, le scripteur affronte donc bien la multiplicité indéfinie des phrases que Benveniste évoque - multiplicité dans laquelle il modèle une entité évanouissante.

Ce choix dans l'infini des combinaisons, que le point de vue formel autorise, obéit malgré tout à des contraintes précises sur lesquelles nous souhaitons revenir avant de proposer notre modèle de production écrite. Tout d'abord, la plasticité langagière - notamment expansive - que le scripteur travaille est en fait limitée par les capacités de la mémoire immédiate : «D'un point de vue formel, la récursivité est théoriquement infinie. En fait, l'usage montre qu'elle est limitée, et les lon-

(21) R. Barthes, La Préparation du roman I et II. Cours et séminaires au Collège de France (1978-1979 et 1979-1980), Seuil / IMEC, $2003: 135$, note 3.

(22) On distinguera la génétique scénarique des grands ensembles structurels (ou macro-génétique) et la génétique scriptique (micro-génétique) qui touche précisément à la textualisation et donc au plan phrastique et stylistiques, les corrections visant à la cohésion de la matière textuelle intervenant après la genèse microstructurelle. 
gues phrases de Proust en constituent un exemple-limite [...] si le prédicat d'une proposition (comme le jour où...) apparaît après une trop longue succession de propositions enchâssées, le lien syntaxique entre les deux termes risque d'être perdu et difficilement récupérable, même à l'écrit » ${ }^{(23)}$. Ensuite, si l'on considère - après Benveniste - que la verbalisation représente la décomposition séquentielle d'une pensée, et la projection sur un axe linéaire d'un message guidé par une intention de faire sens, on comprend que celle-ci ne puisse pas se décomposer à l'infini sans risquer de perdre l'unité qui préside à sa structuration, c'est-à-dire le sens intenté. Autrement dit, la verbalisation tente de coller - au plus près possible (y compris dans la rencontre des non-coïncidences du dire ${ }^{(24)}$ - avec la pensée. Et l'on pourrait être tenté de souligner, à l'inverse, que le fait d'écrire met à jour de nouveaux contenus ${ }^{(25)}$. A cette contrainte cognitive s'ajoute bien évidemment le poids des modèles phrastiques (notamment littéraires, qui relèvent du sociolecte) mais aussi des pratiques singulières - ce que l'on veut bien appeler un « tic d'écriture $»-$ ou un idiolecte..

Au terme de ces analyses, on peut finalement proposer un modèle de production écrite qui tente d'articuler des invariants processuels (ajout, suppression, substitution, déplacement) et des différences individuelles ${ }^{(26)}$. Nous postulons donc un modèle commun à tous les individus et nous situons les différences entre les individus au niveau de variations dans les paramètres de ce modèle général ${ }^{(27)}$ :

\begin{tabular}{|c|c|c|c|c|}
\hline \multicolumn{2}{|c|}{$\begin{array}{l}\text { I. Structure phrastique modifiée } \\
\text { (corrections prédicatives) }\end{array}$} & & \multicolumn{2}{|c|}{$\begin{array}{l}\text { II. Structure phrastique inchangé } \\
\text { (syntaxe identique) }\end{array}$} \\
\hline $\begin{array}{l}\text { Configuration } \\
\text { syntaxique } \\
\text { minimale }=\mathrm{A} 1\end{array}$ & $\begin{array}{l}\text { Configuration } \\
\text { syntaxique } \\
\text { étendue }=\mathrm{B} 1\end{array}$ & & $\begin{array}{l}\text { Configuration } \\
\text { syntaxique } \\
\text { minimale }=\mathrm{C} 1\end{array}$ & $\begin{array}{l}\text { Configuration } \\
\text { syntaxique } \\
\text { étendue =D1 }\end{array}$ \\
\hline$\downarrow$ & $\downarrow$ & $\begin{array}{l}\text { Corrections } \\
\text { substitutives }\end{array}$ & $\downarrow$ & $\downarrow$ \\
\hline $\begin{array}{c}\text { Catalyse } \\
\text { (Expansion / } \\
\text { Ajout) }\end{array}$ & $\begin{array}{c}\text { Ellipse } \\
\text { Condensation / } \\
\text { suppression }\end{array}$ & & & \\
\hline $\begin{array}{c}\text { Configuration } \\
\text { syntaxique } \\
\text { étendue = A2 }\end{array}$ & $\begin{array}{l}\text { Configuration } \\
\text { syntaxique } \\
\text { minimale }=\mathrm{B} 2\end{array}$ & & $\begin{array}{l}\text { Configuration } \\
\text { syntaxique } \\
\text { minimale }=\mathrm{C} 2\end{array}$ & $\begin{array}{c}\text { Configuration } \\
\text { syntaxique } \\
\text { étendue = D2 }\end{array}$ \\
\hline
\end{tabular}

(23) S. Robert, Ibid.

(24) J. Authier-Revuz, 1995.

(25) C'est au reste ce que les psychologues cognitivistes désignent par «effet épistémique ».

(26) Nous suivons en cela le déplacement opéré par A. Culioli (1990:15) de la notion d'universaux à celle d'invariants : "Il faut donc bien qu'il y ait un certain nombre de propriétés communes pour que nous puissions acquérir des systèmes linguistiques équivalents. On posera que parmi l'ensemble des phénomènes que j'ai appelés plus haut «configurations spécifiques » il existe un sous-ensemble qui, effectivement, peut être ramené à un certain nombre de catégories, de schémas, de relations, de termes primitifs, d'opérations, d'enchaînement d'opérations qui vont nous permettre de dégager des invariants que l'on retrouve, sous-jacents à l'activité de langage, quelles que soient les langues que l'on considère $\gg$.

(27) A ce titre, voir J. Lautrey, 2002. 
Cette première version de notre modélisation appelle bien sûr des améliorations ainsi que des confrontations à la variété des corpus, mais ce modèle permet, comme tout modèle, de prévoir les différentes dynamiques scripturaires au niveau phrastique. Surtout, il autorise d'aborder les figures - la catalyse, l'ellipse comme des processus qui, comme l'illustre notre modèle, impliquent des pratiques scripturaires caractéristiques de chaque scripteur ${ }^{(28)}$. Ainsi, sur la base des quatre invariants processuels que sont les quatre opérations d'écriture, il y aurait tendanciellement et parfois successivement (Flaubert, Noël...) des scripteurs - et des écritures - catalytiques ou ajouteuses (1'ajout intervenant sur une base syntaxique brève ou non, soit $\mathrm{A} 1 \Rightarrow \mathrm{A} 2$ ) ou plutôt paradigmatiques (substitutions intervenant sur $\mathrm{C} 1 \Rightarrow \mathrm{C} 2$, D1 $\Rightarrow \mathrm{D} 2$ mais aussi sur les configurations expansées ou condensées A et B) ou plutôt permutante (déplacements) ou plutôt elliptiques ${ }^{(29)}$.

Avant d'illustrer par quelques exemples les différents types d'écriture envisagés dans cette modélisation, il convient de souligner la distinction à opérer entre l'ajout en tant qu'opération/processus et l'ajout comme phénomène transverse au plan phrastique et textuel indépendamment de la genèse du texte ${ }^{(30)}$. Comme opération d'écriture, l'ajout se signale, dans le manuscrit, par sa place interlinéaire ou en marge. S'il peut coïncider avec des segments langagiers qui ont le statut d' " accessoires syntaxiques » (expansions subordonnées, circonstancielles, adjectivales qui seront signalés comme tels par la typographie), l'ajout peut aussi relever de la catégorie du nom (en couplage notamment ${ }^{(31)}$ ) ou du verbe (coordonné par exemple ${ }^{(32)}$ ) ou bien de la coordination (« Je l'ai cru à un moment [mais], comme je viens de le rappeler, j'ai cru bien d'autres choses » $\left.{ }^{(33)}\right)$. L'ajout génétique est donc une opération - qui ne préjuge en rien dans le texte final de son signalement (et statut) initialement secondaire dans le processus d'écriture ${ }^{(34)}$. Il convient également de ne pas perdre de vue l'amplitude textuelle de l'ajout (ou de la condensation/ellipse), c'est-à-dire le niveau de l'analyse linguistique exploré. Partant du principe souligné par Benveniste selon lequel « nous pensons par phrases ", nous privilégions d'abord le plan intra-phrastique, et l'infini théorique des possibles de la phrase de façon à observer le «modelage étroit de la pensée » qui fait de chaque phrase une « entité évanouissante». Cependant, le plan inter-phras-

(28) C'est dans cette optique que Barthes distingue les « esprits à catalyse » et les « esprits paradigmatiques ». Ceci est en accord avec sa conception du style, "langage autarcique qui ne plonge que dans la mythologie personnelle et secrète de l'auteur » (Le Degré zéro de l'écriture) qui relève de ce qu'on appelle « style d'auteur»

(29) Ces tendances varient non seulement d'un scripteur à un autre mais d'une œuvre à l'autre, en particulier en fonction de cette détermination que représente - s'ajoutant aux déterminations de la langue - le genre de discours, plutôt narratif, dialogique ou poétique. Le genre de l'autoportrait, dominant dans l'écriture de Leiris, pose ainsi des problèmes spécifiques notamment dans le rapport oral(ité) / écrit que nous n'abordons pas ici.

(30) A ce titre, voir A. Herschberg Pierrot, "Ajout génétique et ajout linguistique », dans Figures d'ajout. Phrase, texte, écriture (dir. J. Authier-revuz et M.-C. Lala), 2002 : 34-38. On précisera que l'expansion relève d'une acception plus spécifiquement grammaticale, qui comprend à la fois les syntagmes adjoints au sens strict (comme les expansions circonstancielles) et les syntagmes régis (comme les expansions subordonnées et adjectivales).

(31) Voir S. Bikialo, 2003, et «La Nomination multiple : un compromis à la non-coïncidence des mots et de la sensation ", dans Nathalie Sarraute. Du tropisme à la phrase (dir. A. Fontvieille et Ph. Wahl), P.U. Lyon, 2003.

(32) «Des mots, en tirer des idées, et du moins quant à ceux qui se trouvaient ainsi élus, et manipulés, [dégager leur arôme et] ce que j'appellerai leur radiance », Manuscrit de Langage Tangage, $\mathrm{f}^{\circ} 35$, nous signalons 1 'ajout par les crochets.

(33) Manuscrit de Langage Tangage, f72.

(34) Voir aussi S. Pétillon, 2002. 
tique et textuel doit aussi être envisagé. Si, pour un auteur comme Leiris, l'ajout intervient à tous les niveaux ${ }^{(35)}$, on peut distinguer chez Céline, dans son travail d'écriture, une insistance sur 1'ellipse à un niveau macrostructurel - « Ça fait 80.000 pages pour arriver à faire 800 pages de manuscrit, où le travail est effacé » ${ }^{(36)}-$, l'analyse précise et à un niveau microstructurel témoignant plutôt d'une démarche catalytique, comme va le montrer l'exemple à suivre.

\section{A1 > A2 : Ecritures catalytiques / " ajouteuses"}

Appartiennent à ce type d'écriture, l'écriture de M. Leiris, comme nous le montrerons ensuite, mais aussi de Proust, de Céline, de Bazac, dont R. Debray-Genette évoque «l'expansion stellaire, parfois chaotique », où « les repentirs, les interpolations, les additions peuvent étoiler les épreuves » ${ }^{(37)}$, ou encore de Flaubert. Selon A. Albalat, «Flaubert écrit par surcharges. D'abord quelques notes indiquant les idées d'un paragraphe. Il reprend ensuite, il développe, la phrase s'étend, s'épanouit $»{ }^{(38)}$. Comme 1'écrirait Leiris (voir infra), Flaubert arrondit donc la « bedaine » de son texte par ses ajouts ${ }^{(39)}$. Contentons-nous d'un exemple.

On sait qu' on ne dispose que de quelques pages du manuscrit du Voyage au bout de la nuit, mais selon $\mathrm{H}$. Godard « le manuscrit attesté fait apparaître une réalisation en deux temps, puisqu'il est composé de la dactylographie d'un premier état, elle-même abondamment corrigée et enrichie. [...] le mouvement général de passage du premier état à la version publiée consiste en une amplification que l'on peut mesurer à l'échelle du manuscrit (qui passe de 534 feuillets dactylographiés à un total de 899 pages), aussi bien que dans le détail. » ${ }^{(40)}$ Si l'on s'appuie sur la page manuscrite de la tirade de Madelon dans le Voyage, on lit la première version suivante :

« Je te dégoûte! Tu bandes pas comme un autre tu dis quand tu me baises! Tu bandes pas dis dégoûté ? Et lui donc là il jouit pas chaque fois qu'il peut me peloter le dégueulasse » ${ }^{(41)}$.

Dès le manuscrit, des ajouts surtout supra-linéaires interviennent, ainsi que dans le texte publié, pour s'arrêter à la version suivante :

«"Dis-le moi là en français, devant eux, pourquoi que je te dégoûte à présent? Tu

(35) On le montrera à un niveau microstructurel dans la III ${ }^{\text {ème }}$ partie. A un niveau macrostruturel, nous renvoyons à l'analyse des manuscrits de L'Âge d'homme, publiés par C. Maubon («De Lucrèce, Judith et Holopherne à L'Âge d'homme ou comment recoller la tête d'Holopherne », Genesis, 11, 1997) qui témoignent de cette catalyse permanente et récursive ; on constate que L'Âge d'homme est issu de trois brèves «notes rédactionnelles » qui ont permis d'écrire Lucrèce, Judith, Holopherne, texte composé de 23 feuillets dactylographiés, repris et développés par des ajouts génétiques et quasiment sans ratures, sans corrections diminutives.

(36) L.-F. Céline, « Ma grande attaque contre le Verbe» (1957), repris dans Le Style contre les idées, Complexe, $1987:$ 67-68.

(37) R. Debray-Genette, $1977: 27$.

(38) A. Albalat, Le Travail du style enseigné par les corrections manuscrites des grands écrivains, 1903, Armand Colin, 1991: 70.

(39) Notons toutefois que, selon Albalat, le travail s'opère en deux temps chez Flaubert, et qu'à ce mouvement catalytique d'expansion fait souvent suite un mouvement de « condensation » (Ibid. : 89).

(40) H. Godard, Voyage au bout de la nuit de L.-F. Céline, Gallimard, "Foliothèque », 1991 : 162 .

(41) Ibid: 163. 
bandes pas donc comme les autres, dis gros salaud quand tu fais l'amour? Tu bandes pas alors hein ?... Ose le dire là ici ?... Devant tout le monde que tu bandes pas ?..." Malgré sa fureur ça portait un peu à rire la manière dont elle se défendait avec ses remarques. Mais j'ai pas eu le temps de rigoler longtemps, parce qu'elle est revenue à la charge. "Et lui, donc là, qu'elle a fait, il en jouit pas chaque fois qu'il peut m'attraper dans un coin! Ce dégueulasse ! Ce peloteur, qu'il ose donc venir me dire le contraire ?..." » ${ }^{(42)}$

On le voit le texte s'accroit essentiellement par des ajouts d'adverbes de phrase (« donc »), par des expressions relevant de ce style oral que revendiquait Céline ( "Dis-le moi là en français ») ainsi que par un segment de récit entre les deux sections de discours direct. Les différentes positions syntaxiques sont ainsi saturées pour signifier cette « charge » verbale de Madelon.

\section{B1 > B2 : Ecritures elliptiques}

Précisons tout d'abord que l'ellipse est ici pensée en termes dynamiques, de processus de réduction ou de condensation, ce qui implique de ne pas la réduire à la figure de rhétorique telle que la définit I. Tamba, insistant sur son caractère figé : «l'ellipse, comme bien d'autres concepts grammaticaux (paraphrase, concession, etc.) appartient au domaine rhétorique, où elle est répertoriée comme $f^{-}$gure de construction. [...] L'ellipse correspond à l'énoncé, remarquable par son incomplétude, plutôt qu'à l'opération d'omission-suppression qui produit un tel énoncé, comme l'atteste la dénomination même d'ellipse qui s'applique à un phénomène statique et $n$ 'a pas en français [...] de verbe apparenté ${ }^{(43)}$. On suivra davantage les remarques de G. Dessons, qui voit dans l'ellipse «plus qu'une figure, un principe de construction » où les mots manquants sont «perceptibles malgré et par leur absence » représentant ainsi une «énonciation négative » ${ }^{(44)}$. Mallarmé relève de cette écriture, lui qui écrivait à E. Lefébure dans une lettre de 1867 : «je n'ai créé mon œuvre que par élimination », mais aussi Chateaubriand ou certaines œuvres de Bernard Noël selon lequel « c'est plutôt dans l'allègement de la masse verbale que quelque chose peut se produire, dans son évidement... Ecrire, c'est faire ce vide ${ }^{(45)}$. Détaillons un seul exemple.

Selon Albalat - qui le loue au nom des idéaux classiques de concision, de clarté, de justesse -

«Chateaubriand écrivait d'abord sans se relire. Il remplissait de petits carrés de papier ; il les empilait ensuite au hasard. Il s'interrompait pour faire quelques pas, reprenait la plume, rassemblait les feuillets et entamait ce qu'il appelait sa refonte. Il changeait les mots, coupait les phrases, supprimait les qui et les que, qu'il appelait, comme plus tard Flaubert, "l'écueil de notre langue" " ${ }^{(46)}$.

Et de fait, dans la préface de la douzième addition d'Atala, Chateaubriand écrit : «j'ai pesé chaque phrase, examiné chaque mot. Le style, dégagé des épithètes qui l'embarrassaient, marche peut-être avec plus de naturel et de simplicité ». Ce mouvement elliptique est ostensible si l'on reprend la comparaison de ces deux

(42) L.-F. Céline, Voyage au bout de la nuit, 1932, Gallimard, "Folio » : 492-493.

(43) I. Tamba, citée par J.-M. Adam, La Linguistique textuelle, introduction à l'analyse textuelle des discours, 2005, Armand Colin : 111.

(44) G. Dessons, 1992 : 14-15.

(45) B. Noël, L'Espace du poème, POL, 1998: 66.

(46) A. Albalat, 1903/1991: 27. 
versions extraites de l'Essai sur les révolutions et de Génie du Christianisme, proposées par A. Albalat :

« De l'autre côté de la rivière, dans une vaste prairie naturelle, la clarté de la lune dormait sans mouvement sur les rayons où elle était étendue comme des toiles. Des bouleaux dispersés çà et là dans la savane, tantôt, selon le caprice des brises, se confondaient avec le sol en s'enveloppant de gazes pâles; tantôt se détachaient du fond de craie, en se couvrant d'obscurité et formant comme des ailes flottantes sur une mer immobile de lumière. "

« Dans une vaste prairie, de l'autre côté de cette rivière, la clarté de la lune dormait sans mouvement sur les gazons. Des bouleaux agités par les brises, et dispersés çà et là dans la savane, formaient des îles d'ombres flottantes sur une mer immobile de lumière. » ${ }^{(47)}$

La structure syntaxique de la phrase reste globalement inchangée, mais quelques permutations syntaxiques et lexicales (faisant varier les différentes métaphores), et surtout un mouvement global de suppression des expansions réduisent considérablement le fragment. L'expansion adjectivale « naturelle » disparaît, le $\mathrm{SN}$ simple « les gazons » se substitue au $\mathrm{SN}$ complexe « les rayons où elle était étendue comme des toiles », et la structure par balancement « tantôt... tantôt » disparaît au profit d'une structure à un élément. Chateaubriand densifie donc son écriture au gré des réécritures dans une démarche qui relève de l'ellipse.

\section{C1 $>$ C2 et D1 > D2 : Ecritures statiques}

On n'entendra pas cette expression au sens d'absence de mouvement mais d'équivalence des masses textuelles d'un état à un autre : les corrections peuvent être notamment paradigmatiques. Selon A. Albalat, on trouve ce type d'écriture chez Mme de Staël, Th. Gautier, G. Sand, Lamartine ou Fontenelle qu'il classe parmi les « improvisateurs » qui « ont peu retouché leur style» citant cette formule de Gautier à l'appui : «pas de ratures... Au bout de ma plume la phrase arrive retouchée déjà, choisie et définitive : c'est dans ma cervelle que les ratures sont faites " ${ }^{(48)}$. On détaillera ce type d'écriture à partir de Bernard Noël dont les carnets du Syndrome de Gramsci ou de La Maladie du sens ${ }^{(49)}$ sont à peine raturés et dont la page de gauche, laissée blanche comme une vaste marge, n'accueille la plupart du temps que quelques variations lexicales. Dans le manuscrit du Syndrome de Gramsci («Carnet 1, p. 63), on peut lire :

« J'appelle "syndrome de Gramsci" la première manifestation d'un cancer de la langue, généralt dissimulé sous la dénomination de "trou de mémoire". Un cancer, vous le savez, est un foisonnement destructeur, une luxuriance, une folie cellulaire ; un cancer de la langue est une folie semblable, ms inverse. Je vous ai parlé d' 1 cratère implosif : c'est 1 plaie dans laquelle, peu à peu, [le corps] se précipite - une plaie sèche, une plaie blanche dévoratrice de toute la substance qu'ordinairement la langue transforme et réhabilite sans arrêt ».

Du manuscrit au texte publié, les différences seront minimes : «c'est une plaie dévorante, une plaie dans laquelle tout le langage peu à peu se précipite, une plaie blanche ». Les différences relèvent de permutations syntaxiques (déplacements) et lexicales (polyptotes) sans que le volume textuel soit affecté.

(47) Ibid. : 30-31.

(48) Ibid. : 295-299.

(49) Manuscrits conservés à la Bibliothèque Doucet, en cours d'inventaire. 
Par ces différents grands types de processus génétiques et stylistiques, c'est, on le voit, différentes conceptions de l'écriture qui sont mises au jour, et qui permettent de rendre compte du style d'une œuvre de manière dynamique. De plus, cette conception dynamique du style nous permet de répondre aux exigences de modélisation qui sont propres aux sciences du langage en proposant un modèle, une typologie des écritures en production. Ce modèle est du reste exportable - et partant plus facilement exemplifié - vers des scripteurs experts non écrivains (on se souvient de l'opposition entre écrivains et écrivants de Barthes), par exemple des essayistes, des journalistes, etc. Nous réservons ces investigations dans une perspective à venir, elle devra sans doute s'élaborer en collaboration avec la psychologie cognitive de façon à mieux définir - grâce aussi au recueil on live des données - le contour des profils de scripteurs que nous venons de déterminer.

\title{
III. Leiris ou l'impossible phrase succincte : un cas typique d'écriture catalytique.
}

\author{
Dans Biffures, Michel Leiris commente avec précision son écriture « catalyti- \\ que » :
}

« Dans chaque phrase que j'agence - ou presque - apparaissent tôt ou tard (surgis d'emblée ou introduits après coup) un nombre variable de mots jouant un rôle de second plan, soit qu'ils répondent à un besoin (de jour en jour plus accablant) de précautions oratoires ou d'attendus circonstanciés, soit qu'ils figurent de simples chevilles dues à mes exigences pour ce qui touche au rythme (puisqu'il s'avère que, même si j'écris en prose, je ne puis m'empêcher d'exprimer ma pensée sous forme de périodes, de groupes de propositions toujours plus ou moins cadencées). [...] il est loisible à qui prendra la peine (voire même à qui ne prendra pas la peine) d'examiner d'un peu près la structure de mes phrases, de les reconnaître, au vrai, pour ce qu'ils sont : des formations parasitaires qui prolifèrent dans tout ce que j'écris, masquant la pensée authentique plutôt qu'ils ne l'aident à se traduire avec plus de précision et se révélant tout compte fait comme une série d'écrans ». ${ }^{(50)}$

Ces «formations parasitaires », ces «écrans » se retrouvent dans la genèse phrastique de Langage Tangage. Un sondage dans le manuscrit montre que sur un ensemble de 563 retouches, 28 seulement correspondent à des déplacements, 208 constituent des ajouts (repérables aux becquets rouges), 327 correspondent à des substitutions dont 216 sont des substitutions donnant lieu à un ajout de texte. Soit 424 ajouts, et aucune suppression. La phrase suivante illustre cette dimension catalytique de l'écriture M. Leiris. Il y est question précisément des « jeux de masques » de l'auteur se reprochant de «ne pas être mêlé à la mêlée » de son époque. On distinguera trois états : l'état 1 se situe au recto du feuillet et relève du premier jet, l'état 2 se situe au verso du feuillet, appelé par un becquet d'insertion au recto, et il comprend à la fois un texte augmenté et des ajouts ponctuels infra- ou supra-linéaires, et l'état 3 correspond au texte tel qu'il est imprimé :

\section{Etat 1}

Jeux effrontés de masques sur fond de sang, de boue et de misère n'est évidemment pas fait pour apaiser mon trouble. ${ }^{(51)}$

(50) M. Leiris, La Règle du jeu I. Biffures, Gallimard, 1948, coll. «L'imaginaire »: 84.

(51) Le manuscrit se trouve à la Bibliothèque Doucet sous la cote : LRS ms 108 [ $\mathrm{f}^{\circ} 18$ recto ]. Nous suivons les codages de la transcription diplomatique résumés par P.-M. de Biasi, $L a$ 


\section{Etat 2}

Jeux effrontés de masques sur fond de sang, de boue et de misère avec d'affreuses résurgences racistes que mille et une argumentations ne parviennent à réduire, de sorte qu'il est aujourd'hui difficile de croire au rôle utile que pourrait jouer notre parole, le tragique embrouillamini dans lequel nous vivons n'est évidemment pas fait pour apaiser mon trouble.

Jeux effrontés de masques sur fond de sang, de boue et de misère avec côté mœurs d'affreuses résurgences racistes (en particulier) que mille et une argumentations par A + B ni appel aux bon sentiments ne parviennent à réduire, de sorte qu'il est aujourd'hui difficile de croire au rôle utile que pourrait jouer notre parole, le tragique embrouillamini dans lequel nous vivons n'est évidemment pas fait pour apaiser mon traeas trouble.

\section{Etat 3}

Jeux effrontés de masques sur fond de sang, de boue et de misère avec, côté mœurs, d'affreuses résurgences (racistes en particulier) que ni argumentation par $\mathrm{A}+\mathrm{B}$ ni appel aux bons sentiments ne parviennent à réduire, de sorte qu'il est aujourd'hui difficile de croire au rôle utile que pourrait jouer notre parole, le tragique embrouillamini dans lequel nous vivons n'est évidemment pas fait pour apaiser mon trouble. ${ }^{(52)}$

De l'état 1 à l'état 3 se déploie «l'esprit catalytique » de M. Leiris, qui sature progressivement sa phrase de ses «formations parasitaires ». Dans 1'état 1, l'agrammaticalité (sujet au singulier-verbe au pluriel) due à la rupture de construction d'une phrase en cours d'élaboration la donne à voir comme suspendue, ouverte à la catalyse. Et de fait, la comparaison avec l'état 3 montre que ce premier état représente l'ouverture et la clôture de la phrase telle qu'elle sera arrêtée dans l'état 3, version publiée. Tout se passe comme si ce premier jet se savait en attente, formulant en une structure syntaxique minimale (phrase nominale en position frontale puis syntagme verbal non raccordé au sujet par l'accord) le "vouloirdire » global de l'énonciateur. D'un état à l'autre la phrase va donc se catalyser, s'arrondir comme une «bedaine » : comme Leiris l'écrit en effet lui-même dans Langage tangage: « j'entasse ici phrase sur phrase, corrige par suppression moins volontiers que par ajout (comme s'il m'importait d'arrondir la bedaine de mon texte) » ${ }^{(53)}$. Au verso (état 2), on distingue deux moments d'écriture : le passage est réécrit entièrement avec le même syntagme nominal initial, expansé par un complément prépositionnel introduit par «avec » qui s'applique très précisément $\mathrm{au}$ « fond de sang, de boue et de misère », SN composé d'un $\mathrm{N}$ et de deux expansions adjectivales, l'une non classifiante ("affreuses »), l'autre classifiante et nécessaire (« racistes ») et d'une expansion par subordonnée relative introduite par «que», cette subordonnée étant elle-même expansée par une conjonctive relationnelle consécutive introduite par " de sorte qu' ", dont le syntagme verbal va donner lieu à une nouvelle expansion relative introduite par «que ", la suite montrant que tout ce qui précède est en fait une apposition nominale (avec en syntagme tête les «jeux effrontés de masques ») au syntagme nominal « le tragique embrouillamini dans lequel nous vivons » qui va devenir le sujet du syntagme verbal qui était présent dès l'état $1:$ «n'est évidemment pas fait pour apaiser mon trouble». On ne peut donc qu'être frappé, par la prolifération des expansions par subordination,

Génétique des textes, Nathan, 2000, p. 64 : la flèche vers le haut indique 1'endroit où se trouve le becquet d'insertion relie 1'état 1 et 1'état 2.

(52) M. Leiris, Langage Tangage, Gallimard, coll. «L'imaginaire », $1985: 88$.

(53) Ibid. : 170-171. 
par leur récursivité, et par la variété des principes d'expansion mis en œuvre. La phrase progresse par amplifications successives et emboîtées les unes dans les autres, qui font de la phrase leirisienne une entité à la fois parfaitement structurée, très hiérarchisée et construite par mouvements de ruptures et de relances permanentes. Ce mouvement d'amplification présente par ailleurs la particularité de créer une interdépendance très forte entre les différents éléments de la phrase. Dans l'approche des opérations d'amplification et des constructions détachées, ces éléments sont en effet considérés comme des « satellites, des constituants flottants » ${ }^{(54)}$. Si les éléments détachés ou subordonnés sont le plus souvent supprimables syntaxiquement, ils entretiennent avec les éléments de l'unité minimale textuelle une relation d'interdépendance sémantique. La saturation phrastique met donc en scène une volonté d'épuisement des places syntaxiques catalysables, qui fait de la phrase de Leiris une phrase en constante attente de son achèvement ou plutôt d'un « supplément ». C'est cette ouverture (vers des expansions marginales et vers les phrases qui suivent) qui distingue la phrase de Leiris de la " période ", modèle rhétorique défini par Furetière (1690) comme une «petite étendue de discours qui contient un sens parfait, et qui ne doit pas être plus longue que la portée ordinaire de l'haleine ${ }^{(55)}$. La notion de période met l'accent sur l'unité thématique et sémantique («sens parfait»), syntaxique (cohésion grammaticale) et rythmique, voire physique ( "portée ordinaire de l'haleine»), unité et clôture à laquelle s'oppose le plus souvent la phrase de Leiris. De fait, si la phrase est catalysable, elle ne se présente pas comme arrivé à un point de «finition » ou en accord avec une « finalité » mais seulement arrivée à un point de «finitude » ${ }^{(56)}$. Dans Sade, Fourier, Loyola (1971), au cours d'une analogie entre la phrase et le corps chez Sade, Barthes revient sur « l'art de la catalyse »: « la phrase (littéraire, écrite) est elle aussi un corps qu'il faut catalyser, en remplissant tous ses lieux premiers (sujet-verbe-complément) d'expansions, d'incises, de subordonnées, de déterminants ; certes, cette saturation est utopique, car rien ne permet (structuralement) de terminer une phrase : on peut toujours lui ajouter un supplément, qui ne sera jamais, en droit, le dernier (cette incertitude de la phrase rendait Flaubert malheureux) » ${ }^{(57)}$. C'est cette « incertitude de la phrase » que révèle l'étude de la genèse du style de $M$. Leiris dans ses phrases catalysées. Entre « péché par excès » et " péché par défaut», M. Leiris chercher à « trouver la voie intermédiaire - intermerdière ? - grâce à laquelle, récusant l'écriture grise digne elle aussi du dépotoir, je dirais ce qui doit être dit ${ }^{(58)}$.

Cette incertitude de la phrase est aussi celle du sujet de l'écriture, comme en témoigne toute la réflexion de Langage Tantage qui porte sur le mode d'écriture en lien avec la vie et la mort, et sur la possibilité d'une écriture autobiographique à la fois ancrée dans le monde et désengagée, déphasée. Comme M. Leiris l'écrit dans son Journal, faisant le lien entre syntaxe et écriture de soi,

« J'emploie souvent dans mes poèmes des articulations de phrases qui ne sont que des trucs et des chevilles ('tandis que...', 'cependant...', 'mais...', etc.) cela va de

(54) J. Gardes-Tamine, Pour une grammaire de l'écrit, Belin, 2004 : 77. B. Combettes parle, dans Les Constructions détachées en français (Ophrys, 1998) de «constituants périphériques».

(55) Furetière cité par J. Gardes-Tamine, op. cit. : 57. La période a toutefois eu d'autres définitions et réalisations, où prolifèrent les expansions marginales. Voir J.-P. Seguin, 1993.

(56) R. Debray-Genette, $1977: 20$.

(57) R. Barthes, Sade, Fourier, Loyola, Seuil, 1971, repris dans Euvres complètes, III, Seuil, $2002: 814$.

(58) M. Leiris, Langage Tantage, op. cit. : 84. 
pair avec les adjectifs à tout faire. Tout cela, il est vrai, est peut-être inhérent à la forme lyrique. » ${ }^{(59)}$.

\section{Conclusion}

Dans la conscience des scripteurs, il y a une évidence du style et de la phrase, qui les rend tout autant incontournables qu'indéfinissables. En mettant en parallèle leur caractère processuel, "évanouissant», et en les abordant à partir des problématiques de l'élasticité du langage (travaillée par Greimas à travers les mécanismes de 1'expansion/condensation) ainsi que de l'opposition entre ellipse et catalyse revisitée par Barthes, nous proposons un modèle de production écrite qui articule les opérations d'écriture définies comme des invariants processuels aux singularités individuelles - ce qui nous permet d'envisager des types d'écriture : écritures tendanciellement catalytiques ou elliptiques quant aux processus, par la confrontation des volumes textuels et des modes d'expansion syntaxique. Ces écritures sont propres à une œuvre plus qu'à un auteur, même s'il est possible, chez certains écrivains de repérer des tendances dans les processus scripturaux (processus plutôt elliptique ou plutôt catalytique) qui n'empêche en rien la variation ou la confrontation des styles comme l'a parfaitement montré J.-P. Seguin à partir de Voltaire ou de M. Jouhandeau ${ }^{(60)}$. Remis en cause en raison de son caractère figé et normatif, le style ainsi conçu retrouve - par l'ouverture à la psycholinguistique et à la génétique textuelle - son élasticité. "Ouverte » en aval du texte, du côté du lecteur par les travaux sur la lecture, l'œuvre apparaît bien ici ouverte en amont. Par les mouvements syntaxiques d'ellipse et de catalyse, c'est bien l'élasticité du style et de la phrase qui sont exhibés, ce que l'exemple privilégié de M. Leiris a pu nous montrer avec cette démarche catalytique qui lui est propre d'arrondissement centripète, de l'intérieur, de la bedaine de la phrase. L'extrait du manuscrit de Langage Tangage qui suit à la fois évoque - thématiquement - et exemplifie - dans ses différents états génétiques - ce gonflement interne, à partir d'un surgissement, d'un « saut» initial, de la phrase, et du style, en attente :

«Objet d'une appréhension nauséeuse qui court en leitmotiv tout au long de ces feuillets, alors que je devrais m'abstenir en homme biencélé aussi bien élevé que son anglophilie de jeunesse et d'âge mûr le lui prescrit de faire vibrer tout bout de champ la corde du pathétique, le saut qui nous fera franchir la rampe pour en fait ne nous mener nulle part est ce dont l'attente effrayée m'atterre et tout à la fois m'anime. » ${ }^{(61)}$

(59) M. Leiris, Journal, « 19 mai $1929 »$, Gallimard : 173. Et de fait, Y. Peyré a évoqué la « dimension du retrait, de l'ajournement, du détour, de la prolifération dilatoire, repoussant toujours, non la réalité d'un portrait qui se nourrit de ce tragique retard, mais la formulation sèchement juridique d'une identité. C'est que l'identité en crise ne peut se dire, ou que, pour se dire, il lui faut une durée si longue, si plénière que cela revient à un inachèvement ; [...] le tout visé (celui de la vérité globale d'un être [...]) le cède à des prolégomènes interminables » («L'ordre du poème », Europe n 847-848, 1999: 32-33).

(60) J.-P. Seguin, $1996: 5$ («Un texte a toujours des styles, successifs et/ou imbriqués ») et 1999 : 63 («ces faisceaux de traits, que je crois constitutifs du style de Marcel Jouhandeau, ensemble de traits que j' aimerais appelle style lapidaire, en convergence et en conflit avec un style d'apparence bavarde ».

(61) Il s'agit des feuillets 111 recto, 124 verso et 111 bis recto de 1'état manuscrit de Langage Tangage (LRS Ms 108), qui se trouvent à la bibliothèque Doucet. Quatre moments d'écriture sont à distinguer : 1 'étape $1:$ style normal, étape $2:$ gras, étape $3:$ italique, étape $4:$ gras italique. 


\section{Bibliographie}

ADAM, J.-M. (2005) : La Linguistique textuelle. Introduction à l'analyse textuelle des discours, A. Colin.

AlBALAT, A. (1903) : Le Travail du style enseigné par les corrections manuscrites des grands écrivains, Armand Colin, 1991.

AUTHIER-REVUZ, J. (1995) : Ces mots qui ne vont pas de soi. Boucles réflexives et non-coïncidences du dire, Larousse, coll. «Sciences du langage ».

Authier-Revuz, J. et Lala, M.-C. (2002) : Figures d'Ajout. Phrase, texte, écriture, Presses de la Sorbonne Nouvelle.

BARTHES, R. (1967) : «Flaubert et la phrase », Nouveaux essais critiques, Seuil.

BenVeniste, E. $(1966,1974)$ : Problèmes de linguistique générale, tomes 1 et 2 , Gallimard, coll. « Tel».

Berrendonner, A. (1990) : «Pour une macro-syntaxe », Travaux de linguistique, 21, pp. 25-36.

BIASI DE, P.-M. (2000) : La Génétique des textes, Nathan, coll. « 128 ».

BIKIAlo, S. (2003) : Plusieurs mots pour une chose. De la nomination multiple au style de Claude Simon, thèse de doctorat, Université de Poitiers, à paraître.

- (2001) : «Stylistique et périodisation de la prose poétique du XX ${ }^{\text {ème }}$ siècle ", dans Le Temps des lettres. Quelles périodisations pour l'histoire de la littérature française du XXème siècle ? (dir. M. Touret et F. Dugast-Portes), P.U. Rennes, 2001, pp. 181-191.

— (2002) : «De la prédication seconde à la prédication multiple », Aspects de la prédication (dir. S. Leroy et A. Nowakowska), Praxiling-Université PaulValéry, Montpellier III, 2002, pp. 141-153.

- (2004) : L'Âge d'homme de Michel Leiris (avec A. Pibarot), Paris, éditions Atlande.

DEBRAY-GENETTE, R. (1977) : «Génétique et poétique : esquisse de méthode », Littérature, 28, pp. 19-39.

BLANCHE-BENVENISTE, C. (1990) : Le Français parlé, CNRS-Editions.

Culioli, A. (1990) : Pour une linguistique de l'énonciation, opérations et représentations, T1, Ophrys.

DESSONS, G. (1992) : «La parole hantée : épistémologie linguistique de l'ellipse », dans Ellipses, blancs, silences (dir. B. Rougé), PU Pau, pp. 13-22.

FAYOL, M. (1997) : Des idées au textes. Psychologie cognitive de la production verbale, orale et écrite, PUF.

FoucAult, M. (1969) : L'Archéologie du savoir, Gallimard.

GARdes-TAmine, J. (2004) : Pour une grammaire de l'écrit, Belin, 2004.

GodARD, H. (1991) : Voyage au bout de la nuit de L.-F. Céline, Gallimard, «Foliothèque $»$.

Greimas, A.-J. et COURTÈS, J. (1979) : Sémiotique. Dictionnaire raisonné de la théorie du langage, Hachette.

GRÉSILLON, A. (1994) : Eléments de critique génétique, PUF.

GRUNIG, B.-N. (2002) : « Linguistique et brouillons, dynamiques et synchronisation », Langages, 147, Processus d'écriture et marques linguistique, pp. 113126. 
HERSCHBERG PiERROT, A. (2005) : Le style en mouvement. Littérature et art, Belin.

JENNY, L. (1990) : La Parole singulière, Belin.

LAUTREY, J. (2002) : « Le statut de la variabilité entre les individus en psychologie cognitive », dans Invariants et invariabilité, Editions de la MSH-Paris.

LEBRAVE, J.-L. (1988) : «Processus linguistiques et genèse du texte », De la genèse du texte littéraire, Du Lérot.

LE GOFFIC, P. (2005) : «La phrase "revisitée” », Le Français Aujourd'hui, 148, pp. 55-64.

PÉTILLON, S. (2000) : «Énonciation et ponctuation : éléments pour une genèse des parenthèses dans le manuscrit du Plaisir du texte de Roland Barthes ", dans Texte, revue de critique et de théorie littéraire, $\mathrm{n}^{\circ} 27 / 28$, « L'énonciation : la pensée dans le texte », pp. 219-238.

- (2002) : «Roland Barthes ou l'écriture ramifiée : formes et opérations d'ajout dans le manuscrit du Plaisir du texte », Langages, 147, "Processus d'écriture et marques linguistiques. Nouvelles recherches en génétique du texte $»$, pp. 70-84.

— (2006) : «Intentionnalité et révision à l'épreuve du manuscrit d'écrivain. Genèse de Génitrix de F. Mauriac », Langages, S. Pétillon et F. Ganier (dir.), La révision de texte : outils, méthodes et processus, pp. 26-42.

— (2006) : «Style, critique génétique et modèles rédactionnels : perspectives linguistiques », Corpus et style, Corpus $\mathrm{N}^{\circ}$ 5, pp. 37-74.

- (à paraître) : «De l'archive de la création aux processus cognitifs », La critique génétique, pour quoi faire?, éditions de l'Imec.

- (à paraître) : «Décrochement, prédication et modalités d'énonciation : autour du groupe nominal », Faits de Langue, à paraître 2008.

PÉtillon-Boucheron, S. (2002) : Les Détours de la langue. Etude sur la parenthèse et le tiret double, Peeters.

Robert, S. (2002) : « Modèles linguistiques de production », Production du langage (dir. M. Fayol), Hermès, pp. 67-87.

SEguin, J.-P. (1993) : L'invention de la phrase au XVIIIe siècle, Peeters. — (1996) : «Voltaire et la variation des styles », L'Information grammaticale, 70, pp. 5-10.

— (1999) : «Le style de Marcel Jouhandeau : du bavardage au style lapidaire », dans Vous avez dit "Style d'auteur »? (dir. M. Dereu), PU Nancy, pp. 63-73. 